\title{
Magnesium in the Acute Care Setting
}

\author{
Lew SY Benjamin ${ }^{1}$, Lee YY Chanel ${ }^{1}$, Lam VC Matthew ${ }^{1}$ and Lateef Fatimah ${ }^{2,3,4 *}$ \\ ${ }^{1}$ Year 4 Medical Students, Yong Loo Lin School of Medicine, National University of Singapore, Singapore \\ ${ }^{2}$ Department of Emergency Medicine, Singapore General Hospital, Singapore \\ ${ }^{3}$ Yong Loo Lin School of Medicine, National University of Singapore, Singapore \\ ${ }^{4}$ Lee Kong Chian Medical School, Nanyang Technological University and Duke NUS Graduate Medical School, Singapore
}

*Corresponding author: Lateef Fatimah, Department of Emergency Medicine, Singapore General Hospital, Yong Loo Lin School of Medicine, National University of Singapore, Lee Kong Chian Medical School, Nanyang Technological University and Duke NUS Graduate Medical School, Singapore

\begin{tabular}{|c|c|}
\hline ARTICLE INFO & ABSTRACT \\
\hline $\begin{array}{l}\text { Received: 㓞 June 24, } 2021 \\
\text { Published: 慧 July 07, } 2021\end{array}$ & \multirow{2}{*}{$\begin{array}{l}\text { Magnesium is an important element in our bodily functions and metabolism } \\
\text { However, its use may lack emphasis and evidence as compared to the other elements } \\
\text { such as potassium and calcium. This is often guided by personal experience, physicians } \\
\text { familiarity, and comfort level. In the Emergency Department, magnesium may be used } \\
\text { almost daily for acute conditions such as severe asthma, dysrhythmia such as Torsades } \\
\text { des Pointes, hydrofluoric acid burns and pre-eclampsia/ eclampsia syndrome. Its } \\
\text { other uses in migraine and headache syndromes, exacerbation of chronic obstructive } \\
\text { pulmonary disease and alcohol withdrawal remains patchy and not strongly evidence } \\
\text { based. In these conditions, there is a need to explore further with larger multi-centre } \\
\text { trials whether it should be an adjunct or standard therapy. }\end{array}$} \\
\hline $\begin{array}{l}\text { Citation: Lew SY Benjamin, Lee YY } \\
\text { Chanel, Lam VC Matthew, Lateef Fatimah. } \\
\text { Magnesium in the Acute Care Setting. Bi- } \\
\text { omed J Sci \& Tech Res 37(1)-2021. BJSTR. } \\
\text { MS.ID.005941. }\end{array}$ & \\
\hline $\begin{array}{l}\text { Keywords: Magnesium; Loop of Henle; } \\
\text { Asthma; Torsades Des Pointes }\end{array}$ & $\begin{array}{l}\text { Abbreviations: ED: Emergency Department; CLDN16: Claudin-16; FHHNC: Familial } \\
\text { Hypomagnesemia with Hypercalciuria and Nephrocalcinosis; PPIs: Proton Pump } \\
\text { Inhibitors; PTH: Parathyroid Hormone }\end{array}$ \\
\hline
\end{tabular}

\section{Introduction}

Magnesium is an essential element for almost all biological systems, including humans. It normally exists as the divalent cation, $\mathrm{Mg}^{2+}$. As an ion, magnesium has multiple functions in the human body. It plays a critical role in cellular electrical activity through its transmembrane and intracellular modulatory effects. It is responsible for nerve conduction, gastrointestinal absorption, and ATP activity. It is also important in DNA and RNA metabolism as well as plays a critical role as a cofactor in multiple pathways in the body [1]. Magnesium homeostasis in the human body is regulated by various organ systems. The average daily intake of magnesium is approximately $360 \mathrm{mg}$, of which a third is absorbed in the small bowel through a transport system as well as passive diffusion. In addition to absorption, the small bowel also secretes approximately

$40 \mathrm{mg}$ of magnesium in its secretions. Other areas of magnesium absorption include the large bowel, where approximately $20 \mathrm{mg}$ is absorbed. To maintain homeostasis, the magnesium level should be kept fairly constant. Hence, the excess of $100 \mathrm{mg}$ that is absorbed daily is secreted and regulated by the kidneys [1,2]. At the level of the kidney, approximately $80 \%$ of total plasma magnesium is filtered at the glomerulus, with $15-25 \%$ being passively absorbed at the proximal tubule, and $5-10 \%$ at the distal tubule. The majority is reabsorbed at the thick ascending loop of Henle (60-70\%) [2]. While magnesium has many uses in medicine, this article serves to discuss its uses particularly in the acute care setting such as the emergency department (ED). The most common conditions in which magnesium is used include asthma, certain cardiac arrhythmias, and pre-eclampsia. 


\section{Renal Physiology}

\section{Loop of Henle [3]}

Magnesium reabsorption is primarily passive, driven by the electrical gradient generated from the movement of sodium, potassium, and chloride. The relatively positively charged apical end nearer to the tubular lumen promotes the movement of magnesium towards the negatively charged basolateral end nearer to the peritubular capillary. The movement of magnesium between cells is facilitated by tight junction proteins, specifically claudin-16 (CLDN16) and claudin-19 (CLDN19). These proteins are also responsible for the reabsorption of calcium ions. Therefore, factors affecting magnesium transport arise from alterations through the aforementioned variables. For example, loop diuretics that inhibit $\mathrm{Na} / \mathrm{Cl}$ transport result in decreased magnesium reabsorption, while mutations in CLDN16/19 cause familial hypomagnesemia with hypercalciuria and nephrocalcinosis (FHHNC) [3,4].

Other factors that influence magnesium transport at the loop of Henle include but are not limited to:

- Plasma magnesium concentration

- Plasma calcium concentration

- Hormones (PTH \{parathyroid hormone\}, calcitonin, glucagon, beta-adrenergic agonists)

- $\quad$ Acid-base changes

\section{Distal Reabsorption [5]}

The movement of magnesium from the tubular lumen into the cells of the distal convoluted tubule occurs passively. This is mediated by a channel complex (TRPM6, TRPM7), driven by an electrical gradient that is generated by movement of potassium into the tubular lumen. The mechanisms by which magnesium exits the cells of the distal convoluted tubule into the peritubular capillary have yet to be fully elucidated. However, certain proteins that seem to be responsible for this process include the CNNM2 gene which encodes a protein found on the basolateral surface of the DCT, as well as the gamma subunit of the Na-K-ATPase [1,5]. Factors affecting magnesium transport at the DCT include mutations in TRPM6 which causes hypomagnesemia with secondary hypocalcemia, or mutations in the gamma subunit of the Na-K-ATPase which causes isolated renal hypomagnesemia. Certain diuretics that act distally, such as thiazide diuretics, can be associated with hypomagnesemia when used chronically $[2,4,6]$.

\section{Hypomagnesemia}

As discussed above, there are many causes for hypomagnesemia. In clinical practice, the main causes can be divided into gastrointestinal losses as well as renal losses.

\section{Gastrointestinal Losses}

The main causes of hypomagnesemia related to gastrointestinal losses include depletion from diarrhoea or vomiting, acute pancreatitis, medications such as proton pump inhibitors (PPIs) and rarer genetic disorders related to magnesium absorption. Magnesium depletion is more likely to be observed in diarrhoea as compared to vomiting due to the higher magnesium content in lower tract secretions as compared to upper tract secretions. Other scenarios where hypomagnesemia may be observed include malabsorption syndromes, as well as post-small bowel bypass surgery [6]. Acute pancreatitis causes hypomagnesemia in a similar fashion to the way it causes hypocalcemia, by the saponification of these cations into the salt forms in necrotic fat. Additionally, hypomagnesemia might in turn worsen the extent of hypocalcemia by lowering parathyroid hormone (PTH) secretion [7]. A study that investigated PPI use and its association with hypomagnesemia showed that PPI use was associated with an increased risk of hypomagnesemia (odds ratio 1.54) only if the patients were concurrently on diuretics.

There was no significant association between PPI use and serum magnesium if the patients were not on diuretics. While the exact mechanism has not been fully elucidated, it is thought that PPIs affect passive magnesium absorption across intestinal epithelial cells [8-10]. Genetic disorders can also contribute to gastrointestinal loss of magnesium. Intestinal hypomagnesemia with secondary hypocalcemia is a condition that has shown to have both $\mathrm{X}$-linked recessive as well as autosomal recessive inheritance. The autosomal recessive form is due to mutations in the TRPM6 gene as mentioned above. As the protein coded by this gene acts as an apical magnesium channel and is expressed in both intestinal epithelia and the renal tubules, this condition results in both decreased intestinal magnesium absorption as well as renal magnesium wasting [3,11-13].

\section{Renal Losses}

Extrinsic causes of magnesium losses in urine include certain drugs, alcohol, metabolic imbalances, and diabetes mellitus. Loop and thiazide diuretics may cause hypomagnesemia through inhibition of reabsorption of magnesium, though this is typically not significant as increased proximal reabsorption as discussed earlier would counteract these losses. Other well-known examples of nephrotoxic drugs include aminoglycosides, amphotericin B, cisplatin, and digoxin [10]. Alcohol too has a direct effect on hypomagnesemia by causing tubular dysfunction. However, alcohol toxicity can also precipitate other complications such as pancreatitis and malnutrition, each of which also contribute to hypomagnesemia as discussed above [11]. As calcium and magnesium both use similar transport mechanisms in the loop of 
Henle, conditions that cause hypercalcemia can negatively impact the reabsorption of magnesium. An established mechanism of how this happens is seen when calcium binds to the calcium-sensing receptor (CaSR) in the loop of Henle. This results in downregulation of CLDN16 and CLDN19, which as discussed above are responsible for the movement of magnesium in the loop of Henle $[3,12]$. Rarer causes include familial magnesium wasting, the most common of which is Gitelman syndrome. Other causes include Bartter syndrome, and mutations in the various genes responsible for channels in the magnesium transport physiology [13].

\section{Uses of Magnesium in Acute Care Medicine}

Magnesium derangements are more often overlooked compared to those of sodium, potassium and calcium. Below we present the discussion on the use and importance of magnesium in several acute conditions, which may present to the ED.

\section{Asthma}

Asthma is a chronic reversible airway obstruction caused by airway inflammation, smooth muscle spasm and mucous plugging. Usual initial emergency management includes supplemental oxygen, nebulized short acting beta-adrenergic medication (eg. salbutamol), nebulized muscarinic antagonist (eg. ipratropium) and systemic corticosteroids (eg. prednisolone). Supraphysiologic unbound magnesium produces a short term block of calcium channels which interferes with smooth muscle contraction, inducing bronchodilation. Magnesium ion, due to its effects on calcium, also inhibits the release of acetylcholine from motor nerve terminals, inhibiting histamine release from mast cells and decreasing production of mucus in secretory glands [14]. Magnesium sulfate can be administered either through intravenous form or nebulised form.

Forms of magnesium sulfate can be used in conjunction with the usual treatment [15]. There are various treatment regimens available but most of the time, a loading dose of $1.2 \mathrm{~g}$ $2 \mathrm{~g}$ IV magnesium sulfate is given over 20 minutes. The GINA (Global Initiative for Asthma) Guidelines currently suggest that IV magnesium sulfate is not recommended for routine use but could be beneficial in certain population groups which are generally those with more severe exacerbations [16]. Adding magnesium sulfate to the current standard treatments could be beneficial, especially when the response to the initial management is suboptimal as magnesium sulfate is also a relatively safe drug that is readily available. During this period of the COVID 19 pandemic, the use of nebulized medication must be guided by your local institution policies in view it is potentially an aerosol-generating procedure [17].

\section{Arrhythmias}

Torsades de pointes are a unique form of ventricular arrhythmia (polymorphic ventricular tachycardia), most often precipitated by drugs that prolong the QT interval. These include Class I and III antiarrhythmics, phenothiazines, tricyclic antidepressants, some non-sedating antihistamines, butyrophenones and macrolides. Other precipitants include electrolyte imbalances (hypomagnesemia, hypokalemia), hypothyroidism, bradycardia, and cardiac diseases (acute ischemia, myocarditis) [18]. Magnesium acts on myocardial ion channels, most notably on NaK-ATPase, as magnesium is an important cofactor in all reactions involving ATPase. Hence, Na-K-ATPase function is impaired in hypomagnesemia, which results in the ECG changes and subsequent ventricular arrhythmia $[18,19]$. In the majority of patients, a single bolus of $2 \mathrm{~g}$ magnesium sulfate is sufficient to restore sinus rhythm within 5 minutes. Before the discovery of magnesium sulfate's effectiveness, alternative treatments include transvenous cardiac pacing, or pharmacological interventions like atropine. Magnesium has advantages over the alternative treatments because it is safer, highly effective, and easier to administer quickly in emergency situations $[18,20]$.

In patients with chronic heart failure, hypomagnesaemia has been seen, likely due to increased magnesuria from medium to long term loop diuretic use $[4,5,10]$. Another study demonstrated that magnesium treatment reduced episodes of non-sustained VT, but no change in the risk of adverse events or death [20]. In yet another study enrolling 1068 patients with New York Heart Association Class 3 and 4 heart failure patients, serum magnesium was shown to not be an independent risk factor for both sudden death and all causes of death [21]. Also, thus far there have been no strong link between magnesium and both in-hospital and out of hospital cardiac arrest $[19,20]$.

\section{Preeclampsia}

Pre-eclampsia is a condition in pregnancy characterized by new-onset hypertension and organ dysfunction, typically presenting after 20 weeks of gestation. There is a wide spectrum of presentation that can range from mild hypertension to lifethreatening organ dysfunction. Eclampsia refers to the presence of generalized tonic-clonic seizures in a patient with pre-eclampsia in the absence of any underlying neurologic conditions. Features that suggest a severe presentation of preeclampsia or imminent eclampsia include persistent headache, visual symptoms, epigastric pain, altered mental status, clonus, and abnormal biochemical tests (liver function test, full blood count). If left untreated, preeclampsia can progress and lead to complications such as intracerebral hemorrhage, disseminated intravascular coagulation, placental abruption, and death. The only definitive treatment for pre-eclampsia is delivery of the placenta [22]. The MAGPIE trial (2002) investigated the use of magnesium sulfate in the prophylaxis of eclampsia and concluded that the administration of magnesium sulfate in women with pre-eclampsia reduced the risk of developing seizures subsequently, with the number needed to treat being 100 in women without severe features, and 60 in women with severe 
features. Additionally, magnesium prophylaxis also reduced the risk of maternal death in those without severe features of preeclampsia. There was no difference in neonatal outcome [23].

The mechanism by which magnesium acts as an anticonvulsant is still not fully understood. It is believed that magnesium acts as a NMDA receptor antagonist and blocks the action of excitatory neurotransmitters such as glutamate which is released during seizure activity. By inhibiting the NMDA receptor, the seizure threshold is increased. Other hypotheses include the action of magnesium sulfate to stabilize the membrane by its function as a nonspecific calcium channel blocker and decreasing acetylcholine transmission in motor nerve terminals [22,24]. According to the collaborative eclampsia trial regimen25, a loading dose of $4 \mathrm{~g}$ is given intravenously over 5 minutes, followed by an infusion of $1 \mathrm{~g} /$ hour maintained over 24 hours [25]. Recurrent seizures will be treated with a further dose of 2-4g given over 5 minutes. Magnesium sulfate is also found to be superior to phenytoin and diazepam for the treatment of eclampsia $[22,25]$. Thus, to date, magnesium remains the first line drug and gold standard in the management of pre-eclampsia/ eclampsia.

\section{Other Uses}

The use of magnesium in migraine is because of its effect on serotonin receptor activity. There is also postulation on the effect of magnesium on cerebral vasospasm. Some may use it for prophylaxis, whilst there are suggestions for its use in acute migraine [26,27]. For example, Muskop et al demonstrated relief of acute migraine, tension headache and cluster headache in 32 out of 40 of their patients given IV magnesium [27]. Whilst evidence may be less strong and remain controversial, this is an area to watch for further developments. For its use in alcohol withdrawal, it is linked to the often-observed low magnesium levels in chronic alcoholics, malnutrition, and GI losses [11,28]. Recommendations from the American Society of Addiction Medicine states there is no evidence that magnesium supplementation reduces withdrawal severity, frequency of delirium and seizures [28]. The use of magnesium for hydrofluoric acid burns is based on the observation that it is a potent fluoride ion chelator. There are some anecdotal studies that show it reduces the severity of the burns. Hydrofluoric acid burns cause deep injuries to the skin layers and it has been linked with hypocalcaemia, hyperkalemia and hypomagnesemia as well as death in some severe cases [29,30]. Despite its use in severe asthma, the use of magnesium in chronic obstructive lung disease exacerbation, remains controversial $[31,32]$.

\section{Magnesium Sulfate Toxicity}

In patients with renal impairment, dose adjustment and close monitoring is required to avoid magnesium toxicity. With older patients, the risk also increases, thus the need for surveillance monitoring for those on long term therapy. One positive point on the use of magnesium is that the therapeutic index is relatively wide, which means adverse effects are less common. While generally safe, features of magnesium toxicity include its effects on the central nervous system as well as the cardiovascular system. The toxicity is directly correlated with the serum concentration. In order of ascending magnesium concentration, features include loss of deep tendon reflexes ( usually at Mg levels of 3.5-5.0 mmol/l), respiratory paralysis (at Mg levels 5.0-6.5 mmol/l), cardiac arrhythmias and cardiac arrest (>7.5 mmol/l levels) [33]. The management of magnesium toxicity is with calcium gluconate, which is given to patients presenting with cardiac side effects. Furosemide can be given as well to increase the renal excretion of magnesium [33].

\section{Conclusion}

Magnesium is a vital electrolyte that is necessary for the proper functioning of ATP, which in itself is involved in practically every important biochemical process within the human body. In torsades de pointes and pre-eclampsia, the role of magnesium has been well documented to show efficacy and its usefulness in improving patient outcomes. With asthma, the role of magnesium is as second line, in severe attacks not responsive to first line treatment. For other clinical use, there remains some controversies and the use tend to be guided by familiarity with the drug. Currently, the range of very discordant publication certainly calls for a need to have a good, large scale randomized controlled multi-centre trial that will help strengthen the evidence [34,35].

\section{References}

1. Martin KJ, González EA, Slatopolsky E (2008) Clinical Consequences and Management of Hypomagnesemia. Journal of the American Society of Nephrology 20(11): 2291-2295.

2. Hansen BA, Bruserud O (2018) Hypomagnesemia in critically ill patients. Journal of Intensive Care 6: 21.

3. Mount DB (2014) The Loop of Henle. Clinical Journal of the American Society of Nephrology 9(11): 1974-1986.

4. Curry JN, Yu AS (2018) Magnesium Handling in the Kidney. Advances in Chronic Kidney Disease 25(3): 236-243.

5. Subramanya AR, Ellison DH (2014) Distal Convoluted Tubule. Clinical Journal of the American Society of Nephrology 9(12): 2147-2163.

6. Tong GM, Rude RK (2005) Magnesium Deficiency in Critical Illness. Journal of Intensive Care Medicine 20(1): 3-17.

7. Ryzen E, Rude RK (1990) Low intracellular magnesium in patients with acute pancreatitis and hypocalcemia. The Western Journal of Medicine 152(2): $145-148$

8. Danziger J, William JH, Scott DJ, Lee J, Lehman LW, et al. (2013). Proton-pump inhibitor use is associated with low serum magnesium concentrations. Kidney International 83(4): 692-699.

9. Thongon N, Krishnamra N (2011) Omeprazole decreases magnesium transport across Caco-2 monolayers. World Journal of Gastroenterology 17(12): 1574-1583

10. Shah GM, Kirschenbaum MA (1991) Renal magnesium wasting associated with therapeutic agents. Mineral and Electrolyte Metabolism 17(1): 58-64 
11. Elisaf M, Merkouropoulos M, Tsianos EV, Siamopoulos KC (1995) Pathogenetic mechanisms of hypomagnesemia in alcoholic patients. Journal of Trace Elements in Medicine and Biology 9(4): 210-214.

12. Alan S L Yu (2021) Hypomagnesemia: Causes of hypomagnesemia. UpToDate.

13. Viering D, De Baaij J, Walsh SB, Kleta R, Bockenhauer D (2017) Genetic causes of hypomagnesemia, a clinical overview. Pediatric Nephrology 32(7): 1123-1135.

14. Irazuzta JE, Chiriboga N (2017) Magnesium sulfate infusion for acute asthma in the emergency department. Jornal de Pediatr 93(1): 19-25.

15. Shan Z, Rong Y, Yang W, Wang D, Yao P, et al. (2013) Intravenous and nebulized magnesium sulfate for treating acute asthma in adults and children: a systematic review and meta-analysis. Respiratory Medicine 107(3): 321-330.

16. (2021) Global Initiative for Asthma. Asthma Management and Prevention. A guide for health professionals.

17. World Health Organization. Infection prevention and control.

18. Banai S, Tzivoni D (1993) Drug therapy for torsade de pointes. Journal of Cardiovascular Electrophysiology 4(2): 206-210.

19. Bashir Y, Sneddon JF, Staunton HA (1993) Effects of long term oral magnesium chloride replacement in congestive heart failure secondary to CAD. Am J Cardiol 72(15): 1156-1162.

20. Ceremuzynski L, Gebalska J, Wolk R, Makowska E (2000) Hypomagnesimia in heart failure withcentricular arrhythmias. Beneficial effects of magnesium supplementation 247(1): 78-86.

21. Eichhom EJ, Tandon PK, DiBianco R, Timmis GC, Fenster PE, et al. (1993) Clinical and prognstic significance of serum magnesium cncentration in patients with severe congestive heart failure: The PROMISE Study. J Am Coll Cardiol 21(3): 634-640.

22. (2020) Gestational Hypertension and Preeclampsia: ACOG Practice Bulletin, Number 222. Obstetrics and gynecology 135(6): e237-e260.

23. (2007) Magpie Trial Follow-Up Study Collaborative Group. The Magpie Trial: a randomized trial comparing magnesium sulphate with placebo for pre-eclampsia. Outcome for women at 2 years. BJOG 114(3): 300309.

\section{ISSN: 2574-1241}

DOI: 10.26717/BJSTR.2021.37.005941

Lateef Fatimah. Biomed J Sci \& Tech Res

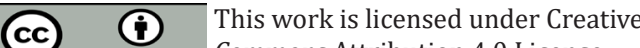
Commons Attribution 4.0 License

Submission Link: https://biomedres.us/submit-manuscript.php
24. Euser AG, Cipolla MJ (2009) Magnesium sulfate for the treatment of eclampsia: a brief review. Stroke 40(4): 1169-1175.

25. Lelia Duley (1995) Which anticonvulsant for women with eclampsia? Evidence from the Collaborative Eclampsia Trial. Lancet 345(8963): 1455-1463.

26. Demirkaya S, Vural O, Dora B, Topçuoğlu MA (2021) The efficacy of Intravenous magnesium sulphate in the management of migraine attacks. Headache 41(2): 171-177.

27. Mauskop A, Altura BT, Cracco RQ Altura BM (1996) Intravenous magnesium sulphate alleviates headache of all types rapidly. Headache 36(3): 154-160.

28. Mayo Smith F (1997) Pharmacological management of alcohol withdrawal: a meta- analysis and evidence-based practice guidelines. JAMA 278(2): 144-151.

29. Kirkpatrick J, Enion D, Burd D (1995) Hydrofluoric acid burns: a review. Burns 21(7): 483-493.

30. Cox RD, Osgood KA (1994) Evaluation of IV magnesium sulphate for the treatment of hydrofluoric acid burns. Clin Toxicol 32(2): 123-136.

31. Shivanathan M, Rajapakse S (2014) Magnesium for acute exacerbation of COPD: a systematic review of randomized trials. Ann Thorac Med 9(2): 77-80.

32. Mukerji S, Shahpuri B, Clayton Smith B, Smith N, Armstrong P, et al. (2015) Intravenous magnesium sulphate $s$ an independent treatment in cute exacerbation of COPD: a single centre randomised, double blinded, parallel group, plcebo controlled trial: a pilot study. N Z Med J 128(1425): 34-42.

33. Cavell GF, Bryant C, Jheeta S (2015) Iatrogenic magnesium toxicity following intravenous infusion of magnesium sulfate: risks and strategies for prevention. BMJ Case Reports 2015: bcr2015209499.

34. Kala J, Abudyyeh A (2017) Magnesium: an overlooked electrolyte. J Emerg Med 52(5): 741-743.

35. Grober U, Schmidt J, Kisters K (2015) Magnesium in prevention and therapy. Nutrients 7(9): 8199-8226.

$\begin{array}{ll}\text { BIOMEDICAL } & \text { Assets of Publishing with us } \\ \text { RESEARCHES } & \text { - Global archiving of articles } \\ \text { - Immediate, unrestricted online access }\end{array}$

